\title{
Entropic wetting in colloidal suspensions
}

\author{
Marjolein Dijkstra ${ }^{1}$ and René van Roij ${ }^{2}$ \\ ${ }^{1}$ Soft Condensed Matter, Debye Institute, Utrecht University, Princetonplein 5, 3584 CC Utrecht, \\ The Netherlands \\ ${ }^{2}$ Institute for Theoretical Physics, Utrecht University, Leuvenlaan 4, 3584 CE Utrecht, \\ The Netherlands
}

Received 3 October 2005

Published 28 October 2005

Online at stacks.iop.org/JPhysCM/17/S3507

\begin{abstract}
We study the adsorption and wetting behaviour of colloidal hard spheres and colloidal rod-like particles at a planar hard wall using computer simulations. Our results show complete wetting by the hard-sphere crystal at the wall-fluid interface, and complete wetting by the nematic phase of the hard-rod fluid at the wall-isotropic fluid interface. In addition, we investigated the effect of polymer-mediated effective interactions on the wetting behaviour of colloidal spheres. The many-body character of the effective colloidal interactions yields an adsorption behaviour that differs enormously from those found for pairwise simple fluids; e.g., far from the triple point, we find three layering transitions in the partial wetting regime prior to a transition to complete wetting by the colloidal liquid.
\end{abstract}

(Some figures in this article are in colour only in the electronic version)

\section{Introduction}

Athermal hard-core fluids have attracted much attention over the years as their phase behaviour is driven by entropy alone. Of course, from thermodynamics we know that a bulk phase transition occurs in a system at constant volume and temperature $T$ if this results in lowering the Helmholtz free energy $F=E-T S$ with $E$ the energy and $S$ the entropy. In most systems, a phase transition occurs because it results in a lowering of $E$ and, at the same time, an increase of $-T S$. However, if we consider athermal systems, i.e., fluids consisting of hard-core interactions, then a phase transition can only occur if this results in an increase of the entropy $S$. The field of entropy-driven phase transitions was pioneered by Onsager in the 1940s, when he showed that a fluid of infinitely thin hard rod-like particles exhibits an isotropic-nematic (IN) phase transition upon increasing the density sufficiently [1]. In addition, computer simulations in 1957 showed a well-defined freezing transition in a fluid of pure hard spheres at a packing fraction well below close packing [2]. These developments showed that purely entropic (packing) effects arising from short-ranged steric repulsions are sufficient to drive phase transitions in the bulk. At first sight, a phase transition towards a more 
structurally ordered phase (configurationally ordered crystal phase or orientationally ordered nematic phase) seems at odds with our intuitive interpretation that the entropy is a measure for the disorder of a system. However, the loss of configurational or orientational entropy can be more than compensated by the increase in entropy that results from the increase in free volume available for each particle. Already many other theoretical/simulation studies, and experimental studies on sterically stabilized colloidal particles have proven that entropydriven phase transitions can occur in many systems.

By contrast the adsorption and wetting behaviour of hard-core fluids near a hard surface is poorly understood. One can enquire, for instance, whether there is complete wetting or partial wetting for hard spheres and for hard rod-like particles. The wetting behaviour of simple fluids is well studied now and it is well known that two scenarios are possible for a liquid droplet in equilibrium with its vapour at a solid substrate. Either the droplet may spread over the surface and form a macroscopically thick liquid film - this corresponds to complete wetting - or partial wetting will occur and a droplet with a certain non-vanishing contact angle will form at the substrate. A thick liquid film is stabilized by the energy of cohesion between the particles and between the particles and the substrate. An interesting question is whether complete wetting or the formation of macroscopically thick films at substrates can be driven by entropy alone. In this paper, we show evidence for complete wetting by hard spheres, by hard rods, and by the colloidal liquid phase of an athermal colloid-polymer mixture at the planar hard-wall interface. Parts of this work have been published elsewhere [3-6].

\section{Colloidal hard spheres}

A contentious issue, which has received much attention, is whether or not there is complete wetting by the hard-sphere crystal at a smooth hard wall. The discussion was instigated in 1992 by Courtemanche and Van Swol, whose molecular dynamics simulations of hard spheres confined between two hard walls showed spontaneous formation of crystalline layers near the wall [7] at a normal pressure below the saturated bulk freezing pressure. The authors concluded that there is complete wetting by the hard-sphere crystal, also called prefreezing, at the wall-fluid interface. However, these findings are disputed as their simulation results for the prefreezing are metastable with respect to capillary freezing: confinement-induced freezing of the whole fluid should occur rather than the formation of only a few crystalline layers at the wall (see [3] and references therein).

In principle, the complete wetting scenario could be confirmed by calculating the contact angle $\theta$, defined by $\cos \theta=\left(\gamma_{\mathrm{wf}}-\gamma_{\mathrm{ws}}\right) / \gamma_{\mathrm{fs}}$, where $\gamma_{\mathrm{wf}}, \gamma_{\mathrm{ws}}$, and $\gamma_{\mathrm{fs}}$ are, respectively, the interfacial tension of the wall-fluid phase at a bulk density $\rho_{\mathrm{b}}=\rho_{\mathrm{f}}$, the wall-crystal interface at $\rho_{\mathrm{b}}=\rho_{\mathrm{s}}$, and the solid-fluid interface at bulk coexistence. The densities of the coexisting bulk fluid and bulk solid phase of hard spheres are $\rho_{\mathrm{f}} \sigma^{3}=0.943$ and $\rho_{\mathrm{s}} \sigma^{3}=1.041$, respectively [2]. A vanishing contact angle corresponds to complete wetting of the wall-fluid interface by the crystal phase. However, using the interfacial tensions from simulations [8, 9], we find $\cos \theta=1.02 \pm 0.37$. Hence, the simulation results are not sufficiently accurate for drawing any definite conclusions.

In order to investigate the adsorption and, in particular, the wetting properties of a fluid, it is important to study a bulk fluid in contact with a single wall. This single-wall system is difficult to treat in simulations, since the fluid in contact with the wall at $z=0$ cannot be treated with periodic boundary conditions in the $z$-direction. In many simulations of adsorption phenomena, the fluid is confined between two identical walls located at $z=0$ and $z=L_{z}$. However, small values of $L_{z}$ can lead to finite size or capillary effects and these may hinder the observation of the single-wall phenomena of interest. In the case of hard spheres, we take 


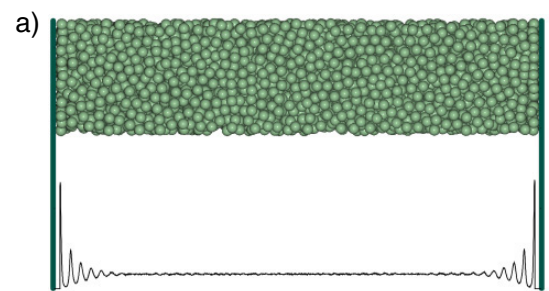

b)

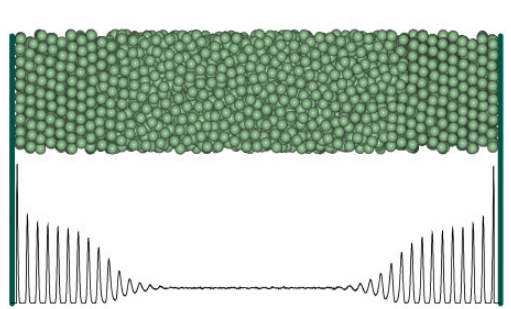

c)

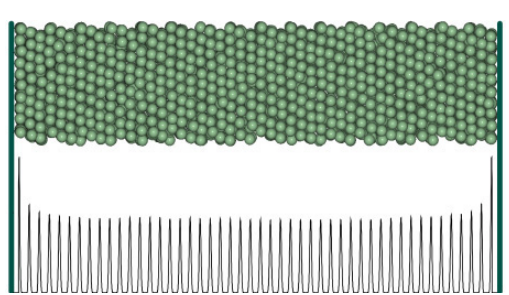

Figure 1. Density profiles $\rho(z) \sigma^{3}$ of hard spheres with diameter $\sigma$ between two planar hard walls at separation $L_{z}=43.5 \sigma$, at bulk densities (a) $\rho_{\mathrm{b}} \sigma^{3}=0.925$, (b) 0.935 , and (c) 1.041, along with typical colloidal configurations.

advantage of the competition between capillary effects and single-wall (wetting) phenomena in planar slits. By making a systematic study of the effect of plate separation, we are able to distinguish a regime where complete wetting of the hard-sphere crystal appears to occur, which is a single-wall phenomenon (i.e. independent of plate separation), from the phenomenon of capillary freezing, which depends on plate separation.

We perform extensive Monte Carlo simulations of pure hard spheres with a diameter $\sigma$ confined between two planar hard walls at different wall separations $L_{z}$. We perform our simulations in the canonical ensemble. We perform simulations at different bulk densities $\rho_{\mathrm{b}}<\rho_{\mathrm{f}}$. Figure 1 shows typical computer configurations along with the corresponding density profiles $\rho(z) \sigma^{3}$ for $L_{z}=43.5 \sigma$ at varying bulk densities $\rho_{\mathrm{b}}$. Figure 1(a) shows the density profile of a fluid with $\rho_{\mathrm{b}} \sigma^{3}=0.925$, while the density profile in figure $1(\mathrm{~b})$ indicates the formation of crystalline layers at the wall. The density profile in the central region of the slit is flat and can be associated with a bulk fluid at $\rho_{\mathrm{b}} \sigma^{3}=0.935$. We observe that the thickness of the portion of the film with crystalline ordering increases with increasing $\rho_{\mathrm{b}}$. The growth of the thickness of the crystalline film can be observed by calculating the adsorption $\Gamma=\int_{0}^{L_{z}}\left(\rho(z)-\rho_{\mathrm{b}}\right)$ for varying $\rho_{\mathrm{b}}$. Figure 2 shows $\Gamma$ as a function of $\rho_{\mathrm{b}}$ for four values of $L_{z}$. We find that the adsorption curves consist of three regimes. (i) At $\rho_{\mathrm{b}} \sigma^{3} \lesssim 0.925$ we find that $\Gamma$ is close to the empirical fit for the adsorption of a fluid of hard spheres obtained in [10]. (ii) For $\rho_{\mathrm{b}} \sigma^{3} \gtrsim 0.925$, the adsorption deviates from this fit due to the formation of crystalline layers at the wall. We observe that $\Gamma$ increases logarithmically as $\rho_{\mathrm{b}}$ increases. We clearly see that all the adsorption curves in figure 2 lie close to each other in this regime. Figure 2 also 


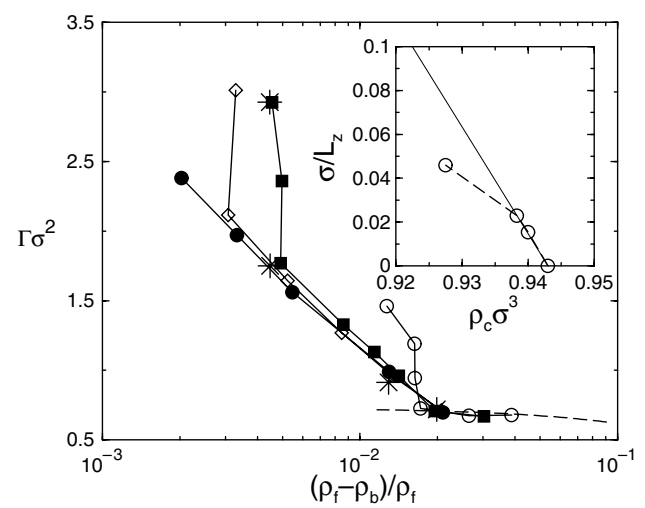

Figure 2. The adsorption $\Gamma \sigma^{2}$ of a fluid of hard spheres in a planar slit versus $\left(\rho_{\mathrm{f}}-\rho_{\mathrm{b}}\right) / \rho_{\mathrm{f}}$ for wall separations $L_{z} / \sigma=21.8(\mathrm{O}), 43.5(\square), 65.21(\triangle), 86.91(\bigcirc)$. For $\rho_{\mathrm{b}} \sigma^{3}<0.925$, the adsorption is close to the empirical fit [10]. The vertical rise in $\Gamma$ found at large $\rho_{\mathrm{b}}$ corresponds to capillary freezing. For intermediate $\rho_{\mathrm{b}}$ the thickness of the wetting crystalline film grows logarithmically. The inset shows the capillary freezing density $\rho_{\mathrm{c}}$ as a function of $L_{z}^{-1}$, obtained from the vertical rise of $\Gamma$. The dashed straight line is the prediction for capillary freezing using the Kelvin equation (1).

shows that the formation of crystalline layers and the logarithmic growth of the film thickness both start at $\left(\rho_{\mathrm{f}}-\rho_{\mathrm{b}}\right) / \rho_{\mathrm{f}} \simeq 0.017$, i.e. $98.3 \%$ of bulk crystallization independent of the plate separation, and can hence be interpreted as a single-wall phenomenon. (iii) At large $\rho_{\mathrm{b}}$, we find a vertical rise in the adsorption, which we associate with capillary freezing induced by the presence of two walls. The bulk density $\rho_{\mathrm{c}}$ at which this jump occurs increases with wall separation and approaches $\rho_{\mathrm{f}}$ from below. In the inset, we plot $\rho_{\mathrm{c}}$ as a function of $L_{z}^{-1}$. We see that $\rho_{\mathrm{c}}$ decreases significantly with decreasing $L_{z}$. In the limit $L_{z} \rightarrow \infty$ the capillary freezing density $\rho_{\mathrm{c}}$ approaches bulk freezing $\rho_{\mathrm{f}}$ linearly in $L_{z}^{-1}$. This linear limiting behaviour can be derived from the Kelvin equation, which reads

$$
\beta\left(\mu_{\mathrm{coex}}-\mu_{\mathrm{c}}\right)=\frac{2 \beta\left(\gamma_{\mathrm{wf}}-\gamma_{\mathrm{ws}}\right)}{\left(\rho_{\mathrm{s}}-\rho_{\mathrm{f}}\right) L_{z}}
$$

where $\mu_{\text {coex }}$ and $\mu_{\mathrm{c}}$ are the chemical potentials at bulk coexistence and at capillary freezing, respectively. Using the Carnahan-Starling expression for the chemical potential of the bulk fluid and using $\rho_{\mathrm{s}} \sigma^{3}=1.041$, and the interfacial tensions obtained from simulations [8], i.e. $\beta \gamma_{\mathrm{wf}} \sigma^{2}=-3.80 \pm 0.18$, and $\beta \gamma_{\mathrm{ws}} \sigma^{2}=-4.37 \pm 0.10$, we find $\left(\rho_{\mathrm{f}}-\rho_{\mathrm{c}}\right) \sigma^{3}=0.2051 \sigma / L_{z}$, denoted by the dashed line in the inset. For $L_{z} / \sigma=43.5,65.21$ we find that the capillary freezing is predicted accurately by the Kelvin equation.

In conclusion, we provide strong evidence for complete wetting by the hard-sphere crystal of the wall-fluid interface as our simulations show the formation of crystalline layers at the wall at $98.3 \%$ of bulk crystallization and the logarithmic growth of the crystalline film, which are both independent of plate separation (single-wall phenomenon) and well separated from capillary freezing that does depend on plate separation. For more details, we refer the reader to [3].

\section{Colloidal hard rods}

We also study the adsorption and wetting behaviour of a fluid of hard spherocylinders with a length-to-diameter ratio $L / D=15$ at a planar hard wall. This model exhibits a 

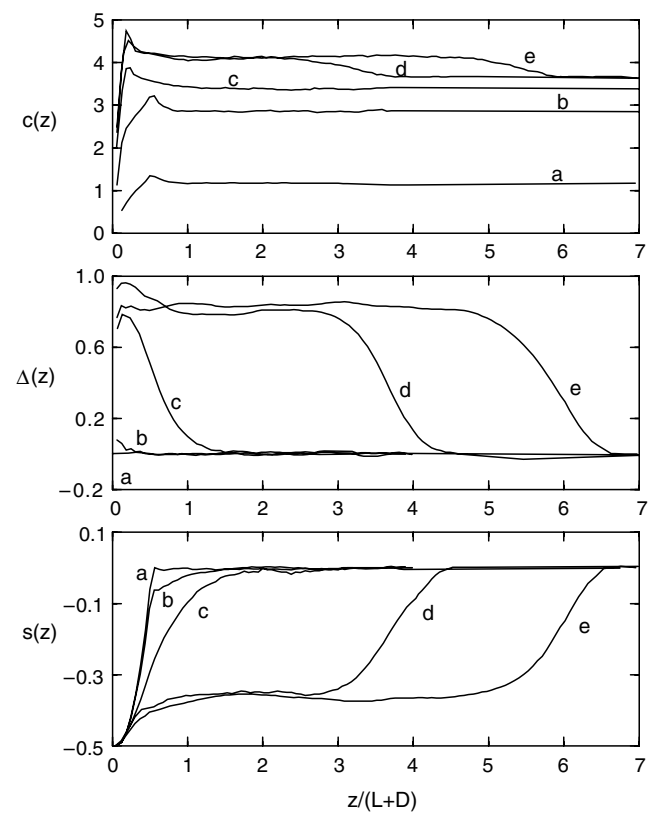

Figure 3. Density and order parameter profiles of a hard-spherocylinder fluid in contact with a single hard wall located at $z=0$. The dimensionless bulk density $c_{\mathrm{b}}=(L+D)^{2} D \rho_{\mathrm{b}}$ far from the wall is (a) 1.169 , (b) 2.855 , (c) 3.379 , (d) 3.633 , and (e) 3.663 . The total density is $c(z)$, the nematic order parameter is $s(z)$, and the biaxial order parameter is $\Delta(z)$.

first-order transition from an isotropic to a nematic phase in the bulk. The coexistence densities are determined by Gibbs ensemble simulations and read $c_{\mathrm{I}}=3.675 \pm 0.003$ and $c_{\mathrm{N}}=4.300 \pm 0.003$, where we define the dimensionless density $c=(L+D)^{2} D \rho$ with $\rho$ the number density. In order to study the wetting behaviour, we developed a Monte Carlo method for simulating fluids in contact with a single wall. A fluid is simulated under the condition that the density far from the wall reaches a bulk density $c_{\mathrm{b}}$ by imposing a penalty function which suppresses large deviations from $c_{\mathrm{b}}[5]$.

Using this method, we measure the density profile and the nematic and biaxial order parameter profiles for varying bulk densities $c_{\mathrm{b}}<c_{\mathrm{I}}$. The density and order parameter profiles are shown in figure 3 for five different values of $c_{\mathrm{b}}$. We discuss first the results for $c_{\mathrm{b}}=1.169$. Figure 3 shows that the density profile $c(z)$ is at a minimum at $z=0$, indicating a depletion of particles close to the wall. $c(z)$ increases with $z$ and reaches a maximum at $z=L / 2$ where there is a kink in $c(z)$. For higher $z, c(z)$ decreases and reaches the bulk value at $z \simeq L$. Turning to the nematic order parameter profile $s(z)$ in figure 3, we find that $s(z=0)=-0.5$ at the wall as the rods have to align with the wall, and increases to 0 for larger $z$, with a kink at $z=L / 2$. The biaxial order parameter profile $\Delta(z)=0$ for all $z$. Thus, we find a uniaxial distribution close to the wall, as $s(z)<0$ and $\Delta(z)=0$, and an isotropic distribution for $z>L$, as $s(z)=0$ and $\Delta(z)=0$. For larger values of $c_{\mathrm{b}}$, we find non-zero values for $\Delta(z)$ close to the wall, corresponding to a biaxial distribution of the rods. The uniaxial-biaxial (UB) transition is estimated to be at about $c_{\mathrm{UB}}=2.80 \pm 0.05$ or $c_{\mathrm{UB}} / c_{\mathrm{I}}=0.76$. Thus, for $c_{\mathrm{b}}<c_{\mathrm{UB}}$ we find uniaxial symmetry for all $z$, whereas biaxiality sets in when $c_{\mathrm{b}}>c_{\mathrm{UB}}$.

If we increase $c_{\mathrm{b}}$ beyond $c_{\mathrm{UB}}$, we clearly observe from the density and the order parameter profiles that a film with in-plane nematic ordering has increasing thickness for increasing $c_{\mathrm{b}}$. 
a)
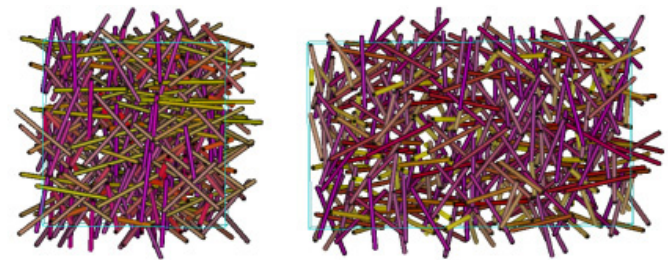

b)
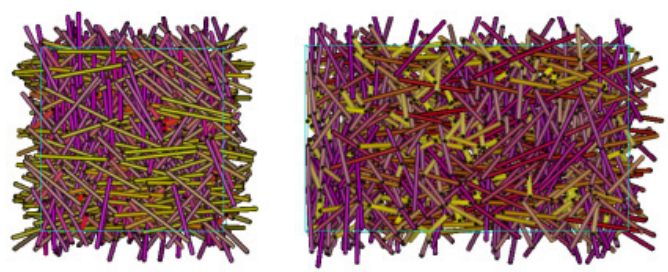

c)
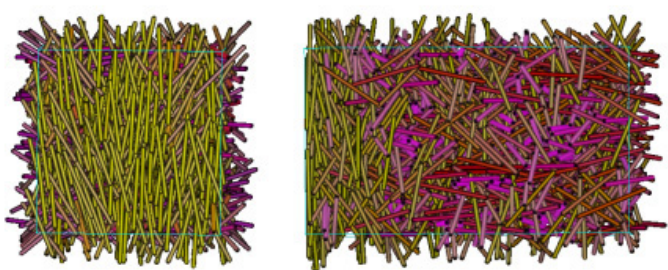

d)
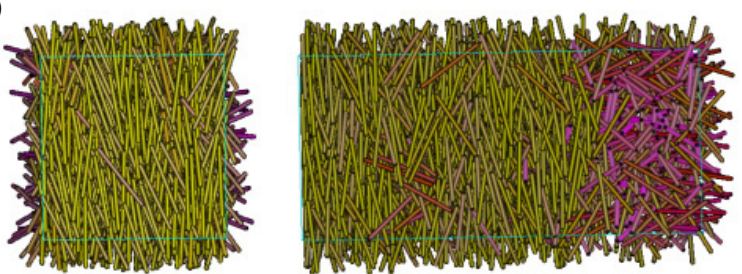

Figure 4. Snapshots of the hard-spherocylinder fluid for varying bulk densities $c_{\mathrm{b}}$ : (a) 1.169 , (b) 2.855 , (c) 3.379, and (d) 3.663. Each left-hand side figure shows a snapshot of the fluid in the $x y$-plane taken from the wall at $z=0$ looking into the fluid, while each right-hand side shows the fluid in the $x z$-plane with the hard wall on the left.

In addition, we find that the adsorption $\Gamma$ increases logarithmically as a function of $\left(c_{\mathrm{I}}-c_{\mathrm{b}}\right) / c_{\mathrm{I}}$. The simulations lend strong support for complete orientational wetting in the limit $c_{\mathrm{b}} \rightarrow c_{\mathrm{I}}$.

More visual information about the ordering at the hard wall is shown in figure 4 where we show snapshots for $c_{\mathrm{b}}=1.169,2.855,3.379$, and 3.633. The left-hand side of each figure shows a snapshot of the fluid in the $x y$-plane taken from the hard wall at $z=0$, while the right-hand side shows the fluid in the $x z$-plane. For $c_{\mathrm{b}}=1.169$ we clearly observe a uniaxial distribution, while at $c_{\mathrm{b}}=2.855$ some small degree of biaxiality has set in. At $c_{\mathrm{b}}=3.379$ and 3.633 we observe that the in-plane nematic film grows steadily, reaching a thickness of about $4(L+D)$ for $c_{\mathrm{b}}=3.633$.

In summary, we find a transition from a uniaxial to a biaxial surface phase, followed, at larger bulk densities, by the formation of a thick nematic film, with the director parallel to the wall, at the wall-isotropic fluid interface. As the density far from the wall $c_{\mathrm{b}}$ approaches the value at bulk isotropic-nematic coexistence $c_{\mathrm{I}}$, the thickness of the nematic film increases logarithmically. The overall adsorption and wetting behaviour is in qualitative agreement with the theoretical prediction for the Zwanzig model [4, 11]. 


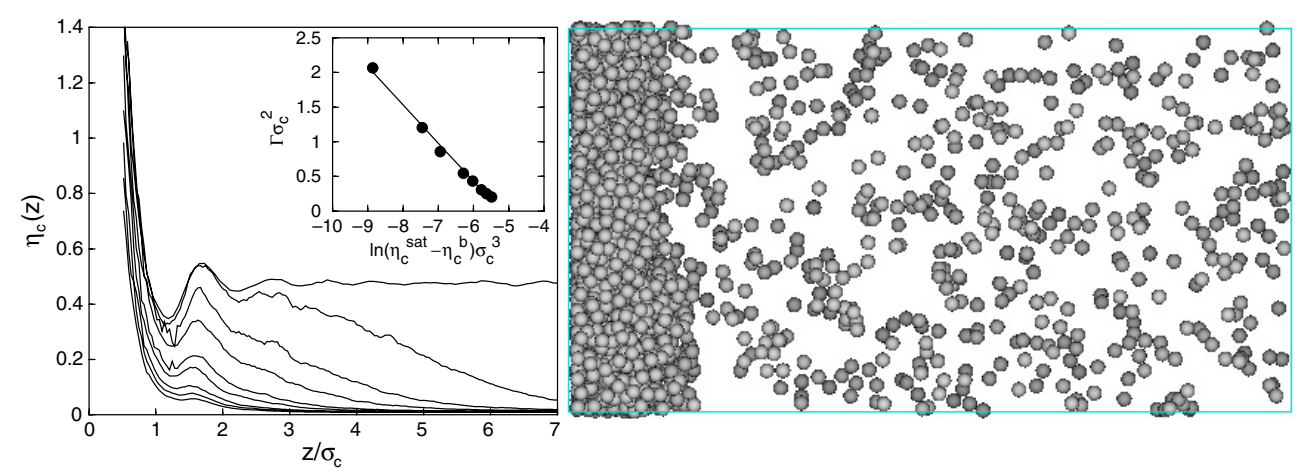

Figure 5. Colloid density profiles $\eta_{\mathrm{c}}(z)$ for reservoir polymer packing fraction $\eta_{\mathrm{p}}^{\mathrm{r}}=1.05$ near a hard wall at $z=0$ (left). Upon approach to gas saturation, the adsorption $\Gamma \sigma_{\mathrm{c}}^{2}$ diverges logarithmically (see the inset), i.e. complete wetting. A typical colloid configuration of a colloidpolymer mixture showing the formation of a thick colloidal liquid film at the colloidal gas-wall interface (right). The polymer coils are not shown.

\section{Colloid-polymer mixtures}

In this section, we study the effect of non-adsorbing polymer on the wetting behaviour of sterically stabilized colloidal spheres. These mixtures can be regarded as one-component systems of hard-sphere colloids with polymer-induced effective interactions of which the strength and the range can be tuned independently by varying the polymer fugacity $z_{\mathrm{p}}$ and the size ratio $q=\sigma_{\mathrm{p}} / \sigma_{\mathrm{c}}$, respectively. Here $\sigma_{\mathrm{p}}$ is twice the polymer radius of gyration and $\sigma_{\mathrm{c}}$ the colloidal hard-core diameter. For sufficiently large $q$ the bulk phase diagram of a colloidpolymer mixture exhibits, at sufficiently high $z_{\mathrm{p}}$, a two-phase region where a colloid-dilute ('gas') and a colloid-dense ('liquid') phase coexist [12-14]. This is similar to the gas-liquid coexistence in simple fluids with $z_{\mathrm{p}}$ playing the role of inverse temperature. However, the cohesion that stabilizes the colloidal liquid phase is not provided by dispersion forces, but by depletion forces generated by the gain of free volume (entropy) for the polymers upon colloidal crowding $[15,16]$. Another difference with simple fluids is that the pairwise additivity of the effective interactions breaks down qualitatively in this $q$-regime, i.e. interactions of three and more bodies are non-negligible. The simplest model that catches the essence of polymerinduced depletion interactions between colloidal hard spheres is the Asakura-Oosawa (AO) model [16]. In this model the polymers are described as non-interacting interpenetrating spheres as regards their mutual interactions, while the colloid-polymer pair interaction is hard-sphere-like such that their distance of closest approach is $\sigma_{\mathrm{cp}} \equiv\left(\sigma_{\mathrm{c}}+\sigma_{\mathrm{p}}\right) / 2$.

By first integrating out the degrees of freedom of the polymer coils in the partition function of the AO model, we derive a formal expression for the exact effective Hamiltonian of the colloids, i.e., it incorporates all the effective many-body interactions $[6,14]$. We then develop a novel Monte Carlo scheme for the exact effective Hamiltonian [6]. Employing a grand canonical version of this scheme, we study the wetting and layering phenomena near a planar hard wall for $q=1$ at fixed polymer fugacity (fixed reservoir polymer packing fraction $\eta_{\mathrm{p}}^{\mathrm{r}}$ ).

We measure the colloid density profile $\eta_{\mathrm{c}}(z)$ at fixed $\eta_{\mathrm{p}}^{\mathrm{r}}$. We use the technique of [5] to impose a flat average profile far away at the wall.

In figure 5, we show the profiles $\eta_{\mathrm{c}}(z)$ in the vicinity of the hard wall for $\eta_{\mathrm{p}}^{\mathrm{r}}=1.05$ at several bulk densities $\eta_{\mathrm{c}}^{\mathrm{b}}<\eta_{\mathrm{c}}^{\text {sat }}$, the saturated colloid gas density. The insets show the 
corresponding dimensionless adsorption $\Gamma \sigma_{\mathrm{c}}^{2}$ as a function of the undersaturation $\ln \left(\eta_{\mathrm{c}}^{\mathrm{sat}}-\eta_{\mathrm{c}}^{\mathrm{b}}\right)$. The formation of a thick liquid film and the logarithmic divergence of $\Gamma \sigma_{\mathrm{c}}^{2}$ with $\eta_{\mathrm{c}} \rightarrow \eta_{\mathrm{c}}^{\text {sat }}$ are strongly indicative of complete wetting for $\eta_{\mathrm{p}}^{\mathrm{r}}=1.05$. For $\eta_{\mathrm{p}}^{\mathrm{r}, \mathrm{c}} \leqslant \eta_{\mathrm{p}}^{\mathrm{r}} \leqslant 1.05$ with $\eta_{\mathrm{p}}^{\mathrm{r}, \mathrm{c}}$ the reservoir polymer packing fraction at the critical point, we also find complete wetting of the colloidal liquid at the wall-gas interface. However, for $\eta_{\mathrm{p}}^{\mathrm{r}} \geqslant 1.10$, the adsorption $\Gamma \sigma_{\mathrm{c}}^{2}$ remains finite for $\eta_{\mathrm{c}}^{\mathrm{b}} \rightarrow \eta_{\mathrm{c}}^{\mathrm{sat}}$, implying partial wetting. This implies that the wetting transition occurs at $\eta_{\mathrm{p}}^{\mathrm{r}, \mathrm{w}}$ with $1.05<\eta_{\mathrm{p}}^{\mathrm{r}, \mathrm{w}}<1.10$

Surprisingly, however, we did find off-coexistence jumps $\delta \Gamma \sigma_{\mathrm{c}}^{2}$ in $\Gamma \sigma_{\mathrm{c}}^{2}$ in three separate regimes $\eta_{\mathrm{p}}^{\mathrm{r}}>\eta_{\mathrm{p}}^{\mathrm{r}, \mathrm{w}}$, i.e. in the partial wetting regime. The jumps can be associated, following [17], with layering transitions. We have no detailed understanding of the mechanism behind these layering transitions, which take place in the partial wetting regime far from the triple point, and not, as in simple fluids, in the complete wetting regime close to the triple point.

In conclusion, we find that the many-body character of the polymer-mediated effective interactions between the colloids yields adsorption phenomena that differ enormously from pairwise simple fluids, e.g. we find three layering transitions in the partial wetting regime, yet well away from the bulk triple point.

\section{Acknowledgments}

It is a pleasure to thank R Evans, J Brader, and M Schmidt for stimulating discussions. This work is part of the research programme of the 'Stichting voor Fundamenteel Onderzoek der Materie (FOM)', which is financially supported by the 'Nederlandse Organisatie voor Wetenschappelijk Onderzoek (NWO)'.

\section{References}

[1] Onsager L 1949 Ann. New York Acad. Sci. 51627

[2] Wood W W and Jacobson J D 1957 J. Chem. Phys. 271207 Alder B J and Wainwright T E 1957 J. Chem. Phys. 271208 Hoover W G and Ree F H 1968 J. Chem. Phys. 493609

[3] Dijkstra M 2004 Phys. Rev. Lett. 93103303

[4] van Roij R, Dijkstra M and Evans R 2000 Europhys. Lett. 49350

[5] Dijkstra M, van Roij R and Evans R 2001 Phys. Rev. E 63051703

[6] Dijkstra M and van Roij R 2002 Phys. Rev. Lett. 89208303

[7] Courtemanche D J and van Swol F 1992 Phys. Rev. Lett. 692078 Courtemanche D J, Pasmore T A and van Swol F 1993 Mol. Phys. 80861

[8] Heni M and Löwen H 1999 Phys. Rev. E 607057

[9] Davidchack R L and Laird B B 2000 Phys. Rev. Lett. 854751

Davidchack R L and Laird B B 2005 Phys. Rev. Lett. 94086102

[10] Henderson J R and van Swol F 1984 Mol. Phys. 51991

[11] van Roij R, Dijkstra M and Evans R 2000 J. Chem. Phys. 1137685

[12] Gast A P, Hall C K and Russel W B 1983 J. Colloid Interface Sci. 96251

[13] Ilett S M et al 1995 Phys. Rev. E 511344

[14] Dijkstra M, Brader J M and Evans R 1999 J. Phys.: Condens. Matter 1110079

[15] Asakura S and Oosawa F 1954 J. Chem. Phys. 221255

[16] Vrij A 1976 Pure Appl. Chem. 48471

[17] Brader J M, Evans R and Schmidt M 2003 Mol. Phys. 1013349 